\title{
LOW FREQUENCY SCATTERING BY A FINITE CONE*)
}

\author{
T. B. A. SENIOR \\ The University of Michigan \\ Dept. of Electrical Engineering \\ Ann Arbor (Michigan) U.S.A.
}

\begin{abstract}
The scattering of a low frequency electromagnetic wave by a metallic cone, whose base is part of a spherical surface centered on the apex of the cone, is analyzed using a mode matching technique. The dipole contributions to the scattering are obtained in complete generality, and numerical results are presented for a wide range of cone angles. Comparisons of the computed data with the predictions of an empirical formula for the scattering reveal both the strengths and weaknesses of the latter.
\end{abstract}

\section{§ 1. Introduction}

At low frequencies the field scattered by a finite body when an electromagnetic wave is incident can be expanded in a series of positive powers of the free space propagation constant $k$, which series is absolutely convergent for sufficiently small $k$. The leading term is produced by the electric and magnetic dipole contributions, and if the incident wavelength $\lambda=2 \pi / k$ is much greater than all the dimensions of the body, the scattered field can be approximated by the leading term alone. The result is Rayleigh scattering for which the scattering cross section is $\sigma=\gamma k^{4}$.

For metallic bodies Siegel [1] has reasoned that $\gamma$ should be proportional to $V^{2}$, where $V$ is the volume of the body, and from an examination of the known expression for the back scattering cross section of a prolate spheroid at axial incidence, he was led to an empirical formula for the constant of proportionality in terms

*) This work was supported in part by the National Science Foundation under Grant GP 9642 . 
of the shape (length-to-width ratio, $\tau$ ) of the body. Thus (see [1]):

$$
\gamma=\frac{4}{\pi} V^{2} F^{2}
$$

with

$$
F=1+\frac{1}{\pi \tau} \mathrm{e}^{-\tau}
$$

The resulting expression for $\sigma$ is most accurate when the body is long and thin $(\tau \gg 1)$, and though the definition of the elongation parameter $\tau$ was later modified $[1,2]$ to produce cross sections in agreement with the known values in such cases as the sphere $(\tau=1)$ and the disc $(\tau=0)$, the formula is still only applicable to the back scattering cross section of a body of revolution at axial incidence.

In practice, however, Siegel's formula is widely used as a means of cross section estimation for any angle of incidence, and because of this, it is desirable to seek some shape of more generality than the spheroid for which the exact Rayleigh cross section can be found. Such a shape should be one for which the electric and magnetic dipole moments can be determined for all angles of incidence and, for preference, be a shape whose low frequency scattering behavior is of interest for its own sake. A body which satisfies these requirements is a finite cone whose base is part of a spherical surface centered on the apex of the cone.

The standard method of solution of low frequency scattering problems is due to Rayleigh [3], and reduces the determination of the scattered field to the solution of certain exterior potential problems for the body in question. The general approach is summarized in section 2 and then particularized to the case of a plane wave at arbitrary incidence on a round backed cone. The potential problems themselves are solved by mode matching (see section 3 ), and as such the method is similar to that used by Schultz et al $[4,5]$ in treating this same geometry for a plane electromagnetic wave at axial incidence. In contrast, however, the boundary value problems which are now encountered are static ones, and because of the considerable simplification which this provides, it is possible to obtain solutions not only for axial incidence on a $30 \mathrm{deg}$. angle cone (as Schultz et al considered), but for all angles of incidence and any cone angle. The numerical results are presented in section 4 . 


\section{§ 2. Formulation}

The general approach to the solution of scattering problems at low frequencies, that is, for $k$ sufficiently small, is to expand the incident and scattered field vectors as power series in $k$, viz.

$$
\boldsymbol{E}^{\mathrm{i}, \mathrm{s}}=\sum_{n=0}^{\infty}(\mathrm{i} k)^{n} \boldsymbol{E}_{n}^{\mathrm{i}, \mathrm{s}}, \quad \boldsymbol{H}^{\mathrm{i}, \mathrm{s}}=\sum_{n=0}^{\infty}(\mathrm{i} k)^{n} \boldsymbol{H}_{n}^{\mathrm{i}, \mathrm{s}} .
$$

When these are substituted into Maxwell's equations and the boundary and radiation conditions, and the coefficients of like powers of $k$ equated, it is found (see, for example, Kleinman [6]) that the conditions on the lowest order scattered fields can be met if

$$
\boldsymbol{E}_{0}^{\mathrm{s}}=\nabla \Phi, \quad \boldsymbol{H}_{0}^{\mathrm{s}}=\nabla \Psi,
$$

where $\Phi$ and $\Psi$ are exterior potentials satisfying Dirichlet and Neumann boundary conditions respectively at the surface of the body. In principle at least, $\Phi$ and $\Psi$ can therefore be determined.

To find the corresponding electric and magnetic dipole contributions to the wave field, we first note that outside some sphere entirely enclosing the scatterer, $\Phi$ and $\Psi$ can be expanded in terms of spherical harmonics in the form

$$
\Phi, \Psi=\sum_{n=1}^{\infty} \frac{Y_{n}(\theta, \phi)}{r^{n+1}},
$$

where $r, \theta, \phi$ are spherical polar coordinates referred to an origin within the body, and for large $r$ the first term in (5) suffices. Moreover, this first term for the function $\Phi$ can be identified as the field of an electric dipole situated at the origin whereas the first term in $\Psi$ is associated with a magnetic dipole. This follows from the fact that the fields due to electric and magnetic dipoles of moments*) $4 \pi \varepsilon_{0} \boldsymbol{P}$ and $4 \pi \boldsymbol{M}$ respectively are

$$
\begin{aligned}
& \boldsymbol{E}=\nabla \wedge \nabla \wedge\left(\boldsymbol{P} \frac{\mathrm{e}^{\mathrm{i} k r}}{r}\right)+\mathrm{i} k \nabla \wedge\left(\boldsymbol{M} \frac{\mathrm{e}^{\mathrm{i} k r}}{r}\right), \\
& \boldsymbol{H}=\nabla \wedge \nabla \wedge\left(\boldsymbol{M} \frac{\mathrm{e}^{\mathrm{i} k r}}{r}\right)-\mathrm{i} k \nabla \wedge\left(\boldsymbol{P} \frac{\mathrm{e}^{\mathrm{i} k r}}{r}\right),
\end{aligned}
$$

*) where $\varepsilon_{0}$ is the permittivity of free space. For brevity, we shall hereafter refer to $\boldsymbol{P}$ and $\boldsymbol{M}$ as the dipole moments. 
(time factor $\mathrm{e}^{-\mathbf{i} \omega t}$ assumed and suppressed), and for $k r$ sufficiently small,

$$
\boldsymbol{E} \simeq-\nabla\left(\frac{\hat{\boldsymbol{r}} \cdot \boldsymbol{P}}{r^{2}}\right), \quad \boldsymbol{H} \simeq-\nabla\left(\frac{\hat{\boldsymbol{r}} \cdot \boldsymbol{M}}{r^{2}}\right) .
$$

Having thereby determined $\boldsymbol{P}$ and $\boldsymbol{M}$, the expressions for $\boldsymbol{E}_{0}^{\mathrm{s}}$ and $\boldsymbol{H}_{0}^{\mathrm{s}}$ at all points exterior to the body can be obtained from (6). In particular, in the far field,

$$
\begin{aligned}
& \boldsymbol{E}_{0}^{\mathrm{S}} \sim \frac{\mathrm{e}^{\mathbf{i} k r}}{r} k^{2}[\hat{\boldsymbol{r}} \wedge(\boldsymbol{P} \wedge \hat{\boldsymbol{r}})+\boldsymbol{M} \wedge \hat{\boldsymbol{r}}], \\
& \boldsymbol{H}_{0}^{\mathrm{s}} \sim \frac{\mathrm{e}^{\mathbf{i} k r}}{r} k^{2}[\hat{\boldsymbol{r}} \wedge(\boldsymbol{M} \wedge \hat{\boldsymbol{r}})-\boldsymbol{P} \wedge \hat{\boldsymbol{r}}],
\end{aligned}
$$

from which the scattering cross section can be deduced.

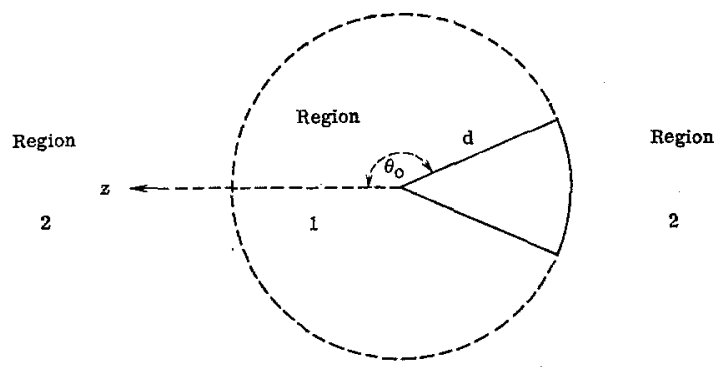

Fig. 1. Cone geometry, showing regions for mode matching.

The specific body to be considered is a round backed cone whose surface is the intersection of a cone of (interior) half-angle $\pi-\theta_{0}$ with a sphere of radius $d$ centered at the apex of the cone (see Fig. 1). The body is assumed perfectly conducting, and in terms of the coordinates $r, \theta, \phi$ with origin at the apex, the surfaces are

$$
\begin{array}{ll}
\theta=\theta_{0}, & 0 \leq r \leq d, \\
r=d, & \pi-\theta_{0} \leq \theta \leq \pi
\end{array} \quad \text { all } \phi .
$$

The incident field is a plane wave of arbitrary polarization incident in an arbitrary direction, and it is convenient to take this as

$$
\begin{aligned}
& \boldsymbol{E}^{\mathbf{i}}=\left(l_{1} \hat{\boldsymbol{x}}+m_{1} \hat{\boldsymbol{y}}+n_{1} \hat{\boldsymbol{z}}\right) \mathrm{e}^{\mathrm{i} k(l x+m y+n z)} \\
& \boldsymbol{H}^{\mathrm{i}}=Y\left(l_{2} \hat{\boldsymbol{x}}+m_{2} \hat{\boldsymbol{y}}+n_{2} \hat{\boldsymbol{z}}\right) \mathrm{e}^{\mathrm{i} k(l \boldsymbol{x}+m y+n z)}
\end{aligned}
$$


where $(x, y, z)$ are rectangular Cartesian coordinates such that

$$
x=r \sin \theta \cos \phi, \quad y=r \sin \theta \sin \phi, \quad z=r \cos \theta
$$

$(l, m, n),\left(l_{1}, m_{1}, n_{1}\right)$ and $\left(l_{2}, m_{2}, n_{2}\right)$ are sets of direction cosines for which

$$
\begin{aligned}
& \left(l_{1}, m_{1}, n_{1}\right)=\left(l_{2}, m_{2}, n_{2}\right) \wedge(l, m, n) \\
& \left(l_{2}, m_{2}, n_{2}\right)=(l, m, n) \wedge\left(l_{1}, m_{1}, n_{1}\right)
\end{aligned}
$$

and $Y$ is the intrinsic admittance of free space.

To the first order at low frequencies,

$$
\begin{aligned}
\boldsymbol{E}^{\mathbf{i}} \sim \boldsymbol{E}_{0}^{\mathbf{i}} & =l_{1} \hat{\boldsymbol{x}}+m_{1} \hat{\boldsymbol{y}}+n_{1} \hat{\mathbf{z}}= \\
& =\nabla\left(l_{1} r \sin \theta \cos \phi+m_{1} r \sin \theta \sin \phi+n_{1} r \cos \theta\right)
\end{aligned}
$$

Since this does not involve the direction cosines $(l, m, n)$ or $\left(l_{2}, m_{2}, n_{2}\right), l_{1}, m_{1}$ and $n_{1}$ can be chosen independently of one another, and for the corresponding scattered electric potential we can therefore write (see (4))

$$
\begin{aligned}
\Phi & =\sum_{n=1}^{\infty} r^{-n-1} \times \\
& \times\left\{l_{1} a_{n}^{(1)} P_{n}^{1}(\cos \theta) \cos \phi+m_{1} a_{n}^{(2)} P_{n}^{1}(\cos \theta) \sin \phi+n_{1} a_{n}^{(3)} P_{n}(\cos \theta)\right\} .
\end{aligned}
$$

valid for $r \geq d$. The coefficients $a_{n}^{(j)}, j=1,2,3$, are independent of $l_{1}, m_{1}$ and $n_{1}$, and in general their determination requires the solution of three elementary potential problems. For a body of revolution, however, the symmetry about the $z$ axis dictates that $a_{n}^{(1)}=a_{n}^{(2)}$, which reduces the number of potential problems to two. In terms of the $a_{n}^{(j)}$, the electric dipole moment is

$$
\boldsymbol{P}=-\left(l_{1} a_{1}^{(1)} \hat{\boldsymbol{x}}+m_{1} a_{1}^{(2)} \hat{\boldsymbol{y}}+n_{1} a_{1}^{(3)} \hat{z}\right) .
$$

For the scattered magnetic field, the procedure is similar in all respects. Corresponding to (10), we have

$$
\boldsymbol{H}^{\mathbf{i}} \sim \boldsymbol{H}_{0}^{\mathbf{i}}=Y \nabla\left(l_{2} r \sin \theta \cos \phi+m_{2} r \sin \theta \sin \phi+n_{2} r \cos \theta\right),(13)
$$


implying (see (4))

$$
\begin{aligned}
\Psi & =Y \sum_{n=1}^{\infty} r^{-n-1} \times \\
& \times\left\{l_{2} b_{n}^{(1)} P_{n}^{1}(\cos \theta) \cos \phi+m_{2} b_{n}^{(2)} P_{n}^{1}(\cos \theta) \sin \phi+n_{2} b_{n}^{(3)} P_{n}(\cos \theta)\right\}
\end{aligned}
$$

valid for $r \geq d$. In general three elementary potential problems must be solved to find the $b_{n}^{(j)}, j=1,2,3$, but for a body of revolution about the $z$ axis, $b_{n}^{(1)}=b_{n}^{(2)}$. In terms of the $b_{n}^{(j)}$, the magnetic dipole moment is

$$
\boldsymbol{M}=-Y\left(l_{2} b_{1}^{(1)} \hat{\boldsymbol{x}}+m_{2} b_{1}^{(2)} \hat{\boldsymbol{y}}+n_{2} b_{1}^{(3)} \hat{\mathbf{z}}\right) .
$$

\section{§ 3. Analysis}

For the round backed cone shown in Fig. 1, there are four independent components of the dipole moments to be computed, and it is convenient to approach the task by considering separately the potential problems generated by four elementary incident fields. If, for example, an incident field is chosen having $l_{1}=1$ with $m_{1}=n_{1}=0$, the transverse electric dipole moment $a_{1}^{(1)}\left(=a_{1}^{(2)}\right)$ can be found; alternatively, if $l_{1}=m_{1}=0$ but $n_{1}=1$, the axial electric dipole moment results; and similarly for the magnetic dipole. All of these four potential problems are comparable as regards the analysis involved, and it is therefore sufficient to detail the procedure for $a_{1}^{(1)}$ alone.

As indicated by (10) and (11) with $l_{1}=1, m_{1}=n_{1}=0$, the total electric potential in $r \geq d$ is

$$
\Phi \equiv \Phi_{2}=\sum_{n=1}^{\infty}\left(r^{-n-1} a_{n}^{(1)}+r \delta_{1 n}\right) P_{n}^{1}(\cos \theta) \cos \phi
$$

where $\delta_{m n}$ is the Kronecker delta function, whereas in region 1 (see Fig. 1) we assume

$$
\Phi \equiv \Phi_{1}=\sum_{\nu} c_{\nu} \nu^{\nu} P_{\nu}^{1}(\cos \theta) \cos \phi
$$

with the coefficients $c_{p}$, as well as the summation variable $\nu$, still to be determined. From the boundary conditions on the electromagnetic field at the surface of the cone and the requirement of 
continuity across $r=d$, we have (for all $\phi$ ):

$$
\left.\begin{array}{rlrl}
\frac{\partial \Phi_{1}}{\partial r} & =\frac{\partial \Phi_{1}}{\partial \phi}=0 & & \text { for } \theta=\theta_{0}, \\
\frac{\partial \Phi_{2}}{\partial \phi} & =\frac{\partial \Phi_{1}}{\partial \phi} & & \text { for } r=d, 0 \leq \theta<\theta_{0} \\
& =0 & & \text { for } r=d, \theta_{0}<\theta \leq \pi \\
\frac{\partial \Phi_{2}}{\partial \theta}=\frac{\partial \Phi_{1}}{\partial \theta} & & \text { for } r=d, 0 \leq \theta<\theta_{0} \\
& =0 & & \text { for } r=d, \theta_{0}<\theta \leq \pi \\
\frac{\partial \Phi_{2}}{\partial r}=\frac{\partial \Phi_{1}}{\partial r} & & \text { for } r=d, 0 \leq \theta<\theta_{0} .
\end{array}\right\}
$$

The first of these conditions can be satisfied by choosing $v$ such that

$$
P_{v}^{1}\left(\cos \theta_{0}\right)=0,
$$

with the summation in (17) extending over all the zeros $\boldsymbol{v}=\boldsymbol{\nu}_{i}$, $i=1,2,3, \ldots$, of the Legendre function of order unity. By invoking the orthogonality of the functions $P_{n}^{\mathbf{1}}(\cos \theta)$ over the range 0 to $\pi$, the second and third boundary conditions give

$$
d^{-m-2} a_{m}^{(1)}+\delta_{1 m}=\frac{2 m+1}{2 m(m+1)} \sum_{\nu} c_{\nu} d^{\nu-1} X_{m \nu}
$$

where

$$
X_{m v}=\frac{1}{m(m+1)} \int_{0}^{\theta_{o}}\left(\sin \theta \frac{\partial P_{m}^{1}}{\partial \theta} \frac{\partial P_{v}^{1}}{\partial \theta}+\frac{P_{m}^{1} P_{v}^{1}}{\sin \theta}\right) \mathrm{d} \theta .
$$

It can be shown [5] that

$$
X_{m \nu}=\left.\frac{1}{m(m+1)-\nu(\nu+1)} \sin \theta_{0} P_{m}^{1}\left(\cos \theta_{0}\right) \frac{\partial P_{v}^{1}}{\partial \theta}\right|_{\theta=\theta_{0}}
$$

providing $v \neq m$. Finally, from the fourth boundary condition we have

$$
\begin{gathered}
\sum_{n=1}^{\infty}(n+1)\left(d^{-n-2} a_{n}^{(1)}-\frac{1}{2} \delta_{1 n}\right) P_{n}^{1}(\cos \theta)=-\sum_{\nu} v c_{p} d^{v-1} P_{\nu}^{1}(\cos \theta) \\
0 \leq \theta<\theta_{0}
\end{gathered}
$$


from which a further relation between the $a_{n}^{(1)}$ and the $c_{p}$ can be found by using the orthogonality [5] of the functions $P_{v}^{1}(\cos \theta)$ over the range $0 \leq \theta<\theta_{0}$. Hence

$$
c_{\nu} d^{v-1}=-\frac{1}{\Lambda_{v}} \sum_{n=1}^{\infty}(n+1)\left(d^{-n-2} a_{n}^{(1)}-\frac{1}{2} \delta_{1 n}\right) X_{n v},
$$

where

$$
\Lambda_{\nu}=\int_{0}^{\theta_{0}} \sin \theta\left\{P_{\nu}^{1}(\cos \theta)\right\}^{2} \mathrm{~d} \theta
$$

and by substituting this expression for $c_{\nu} d^{p-1}$ into $(20)$, we obtain

$$
m A_{m}^{(1)}+\delta_{1 m}=-\sum_{n=1}^{\infty}\left(n+\frac{1}{2}\right) A_{m}^{(1)} \sum_{\nu} \frac{X_{m \nu} X_{n \nu}}{\nu \Lambda_{v}},
$$

$m=1,2,3, \ldots$, with

$$
A_{m}^{(\mathbf{1})}=\frac{m+1}{2 m+1}\left(d^{-m-2} a_{m}^{(1)}-\frac{1}{2} \delta_{1 m}\right) .
$$

The equations (24) constitute an infinite set of simultaneous equations for the determination of the coefficients $A_{m}^{(1)}$. The particular coefficient of interest to us is $A_{1}^{(1)}$ in terms of which the transverse component of the electric dipole moment is

$$
a_{1}^{(1)}=\frac{d^{3}}{2}\left(3 A_{1}^{(1)}+1\right) \text {. }
$$

The other three potential problems are of a similar nature, and in each case an infinite set of equations is obtained from which to compute quantities related to $a_{1}^{(3)}, b_{1}^{(1)}$ and $b_{1}^{(3)}$. Thus, for the axial component of the magnetic dipole moment, the equations corresponding to $(24)$ are

$$
(m+1) B_{m}^{(3)}+\delta_{1 m}=-\sum_{n=1}^{\infty}\left(n+\frac{1}{2}\right) B_{n}^{(3)} \sum_{v} \frac{X_{m v} X_{n v}}{(v+1) A_{v}}
$$

$m=1,2,3, \ldots$, where $v, X_{m}$ and $A_{v}$ have the same definitions as above, and

$$
B_{m}^{(3)}=-\frac{1}{2 m+1}\left(d^{-m-2} b_{m}^{(3)}+\delta_{1 m}\right)
$$

implying

$$
b_{1}^{(3)}=-d^{3}\left(3 B_{1}^{(3)}+1\right)
$$


For the transverse component of the magnetic dipole moment, the summation must be carried out over the zeros of the first derivative of the Legendre function of order unity, so that here $v=\boldsymbol{v}_{i}, i=$ $=1,2,3, \ldots$, with

$$
\left.\frac{\partial}{\partial \theta} P_{\nu}^{1}(\cos \theta)\right|_{\theta=\theta_{0}}=0,
$$

and the resulting set of equations is

$$
m B_{m}^{(1)}+\delta_{1 m}=-\sum_{n=1}^{\infty}\left(n+\frac{1}{2}\right) B_{n}^{(1)} \sum_{v} \frac{X_{m v} X_{n v}}{v \Lambda_{v}},
$$

$m=1,2,3, \ldots$, where $A_{v}$ still has the form shown in (23), but now $X_{m v}=\left.\frac{v /(m+1)}{m(m+1)-v(v+1)} \sin \theta_{0} P_{v}^{1}\left(\cos \theta_{0}\right) \frac{\partial}{\partial \theta} P_{m}^{1}(\cos \theta)\right|_{\theta=\theta_{0}}$

providing $\boldsymbol{v} \neq m$, and

$$
B_{m}^{(1)}=-\frac{m}{2 m}+1 \frac{1}{+1}\left(d^{-m-2} b_{m}^{(1)}+\delta_{1 m}\right) .
$$

The transverse component itself is

$$
b_{1}^{(1)}=-\frac{d^{3}}{2}\left(3 B_{1}^{(1)}+2\right) .
$$

Finally, for the axial component of the electric dipole moment, the required zeros are those of the Legendre function of order zero, that is, $v=v_{i}, i=1,2,3, \ldots$, with

$$
P_{\nu}\left(\cos \theta_{0}\right)=0 .
$$

The equations that result are

$$
(m+1) A_{m}^{(3)}+\delta_{1 m}=-\sum_{n=1}^{\infty}\left(n+\frac{1}{2}\right) A_{n}^{(3)} \sum_{\nu} \frac{X_{m \nu} X_{n v}}{(v+1) \Lambda_{\nu}},
$$

$m=1,2,3, \ldots$, where $A_{v}$ again has the form shown in (23), but

$$
X_{m v}=-\left.\frac{(m+1)(v+1)}{m(m+1)-v(v+1)} \sin \theta_{0} P_{m}\left(\cos \theta_{0}\right) \frac{\partial P_{v}}{\partial \theta}\right|_{\theta=\theta_{0}}
$$


providing $\boldsymbol{\nu} \neq m$, and

$$
A_{m}^{(3)}=\frac{1}{2 m+1}\left(d^{-m-2} a_{m}^{(3)}-\frac{1}{2} \delta_{1 m}\right)
$$

implying

$$
a_{i}^{(3)}=\frac{d^{3}}{2}\left(3 A_{1}^{(3)}+1\right)
$$

We thus have four infinite sets of simultaneous equations from which to compute the dipole moments. All four sets are of rather similar form and are to be solved for a variety of $\theta_{0}$. Each contains a quantity $X_{m}$, whose simplified expression (22), (32) or (37) is valid only if $v \neq m$. Knowledge of the Legendre function zeros shows that this condition is certainly fulfilled if $\theta_{0}>\pi / 2$.

\section{§ 4. Numerical Results}

For any given value of $\theta_{0}$ there are three main computational tasks associated with the solution of each equation set: (i) the calculation of an adequate number of Legendre function zeros; (ii) the evaluation of the various factors involved, including the numerical integration of the expression for $\boldsymbol{A}_{\boldsymbol{\nu}}$; and (iii) the matrix inversion. Only the first of these is other than straightforward, and even here we were fortunate in having available a procedure that had been developed [7] in treating the scattering of an electromagnetic wave by a semi infinite cone [8]. Taking, for example, the problem of the transverse electric dipole moment for which the required zeros are those of the Legendre function of order unity, we write

$$
P_{\nu}^{1}\left(\cos \theta_{0}\right)=-\frac{1}{(\pi)^{\frac{1}{2}} \sin \theta_{0}} \frac{(v+1) !}{\left(v+\frac{1}{2}\right) !} S_{\nu}\left(\theta_{0}\right)
$$

where

$$
S_{\nu}\left(\theta_{0}\right)=\sum_{k=0}^{\infty} \frac{\left(-\frac{1}{2}\right)_{\mathrm{k}}(\nu)_{\mathrm{k}}}{k !\left(v+\frac{3}{2}\right)_{\mathbf{k}}} \sin \left\{(\nu+2 k) \theta_{0}\right\},
$$

valid for $0<\theta_{0}<\pi$. The series does converge, albeit slowly, at a rate which is independent of $\theta_{0}$, and the zeros can be found by an iterative method. Similarly, for the zeros of the Legendre function 
derivative, we use the recurrence relations:

$$
\begin{aligned}
\frac{\partial}{\partial \theta_{0}} P_{\nu}^{1}\left(\cos \theta_{0}\right)=- & \frac{\nu}{(\pi)^{\frac{1}{2}} \sin ^{2} \theta_{0}} \frac{(v+1) !}{\left(\nu+\frac{1}{2}\right) !} \times \\
& \times\left\{\cos \theta_{0} S_{\nu}\left(\theta_{0}\right)-\left(1+\frac{1}{2 \nu}\right) S_{\nu-1}\left(\theta_{0}\right)\right\},
\end{aligned}
$$

and again resort to an iterative method. For the zeros of the zero order function, however, no program was available, but in order to use to the utmost the procedures already developed, the recurrence relations for the Legendre function were employed to give

$$
P_{\nu}\left(\cos \theta_{0}\right)=\frac{1}{\nu(\nu+1)}\left\{\frac{\partial P_{\nu}^{1}}{\partial \theta_{0}}+\cos \theta_{0} P_{v}^{1}\right\} .
$$

With the aid of (40), the right hand side can be expressed in terms of the same function $S_{\nu}\left(\theta_{0}\right)$ previously computed, and the zeros again found by iteration.

The computations were carried out on an IBM 360 computer, and numerical solutions to the sets of equations shown in (24), (27), (31) and (36) were obtained for $\theta_{0}=95 \mathrm{deg}$. through $150 \mathrm{deg}$. (increased with steps of $5 \mathrm{deg}$.) and for $\theta_{0}=150 \mathrm{deg}$. through $177 \frac{1}{2} \mathrm{deg}$. (increased with steps of $2 \frac{1}{2} \mathrm{deg}$.). Only the leading coefficients $A_{1}^{(1)}$, $B_{1}^{(3)}, B_{1}^{(1)}$ and $A_{1}^{(3)}$ were printed out from which the dipole moments were deduced as indicated in (26), (29), (34) and (39), and in each case it was found that a maximum of 20 zeros was adequate to give results accurate to three significant figures. The values of $a_{1}^{(1)}$, $b_{1}^{(3)}, b_{1}^{(1)}$ and $a_{1}^{(3)}$ are plotted as functions of $\theta_{0}$ in Fig. 2.

Having determined the dipole moments, the lowest order electric and magnetic fields can be obtained at all points exterior to the cone by substituting into (6) the expressions for $\boldsymbol{P}$ and $\boldsymbol{M}$ given in (12) and (15) respectively. The far zone back scattered field is of particular interest to us. This follows from (8) on putting $\hat{\boldsymbol{r}}=$ $=-(l, m, n)$, and the direct and cross polarized components are then

$$
\begin{aligned}
& \left.\left(l_{1}, m_{1}, n_{1}\right) \cdot \boldsymbol{E}_{0}^{\mathbf{s}}\right|_{\boldsymbol{r}=-(l, m, n)}=\frac{\mathrm{e}^{\mathbf{i} k r}}{k r} S_{\|}, \\
& \left.\left(l_{2}, m_{2}, n_{2}\right) \cdot \boldsymbol{E}_{0}^{\mathbf{s}}\right|_{r=-(l, m, n)}=\frac{\mathrm{e}^{\mathrm{i} k r}}{k r} S_{\perp}
\end{aligned}
$$




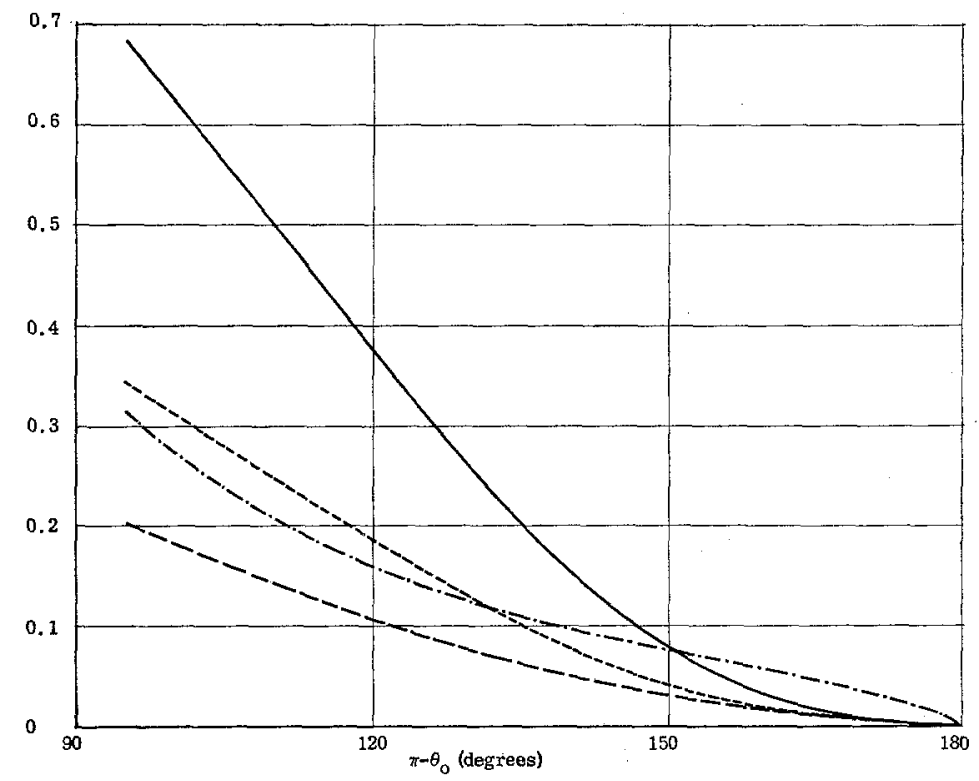

Fig. 2. Computed dipole moments:

$-a_{1}^{(1)} / d^{3},-;-a_{1}^{(3)} / d^{3}, \cdot-\cdot-\cdot-\cdot ; b_{1}^{(1)} / d^{3},----;$; and $b_{1}^{(3)} / d^{3}$,

respectively, where, from (8),

$$
\begin{aligned}
& S_{\|}=k^{3}\left[\left(l_{1}, m_{1}, n_{1}\right) \cdot \boldsymbol{P}-\left(l_{2}, m_{2}, n_{2}\right) \cdot \boldsymbol{M}\right], \\
& S_{\perp}=k^{3}\left[\left(l_{2}, m_{2}, n_{2}\right) \cdot \boldsymbol{P}+\left(l_{1}, m_{1}, n_{1}\right) \cdot \boldsymbol{M}\right] .
\end{aligned}
$$

Substituting from (12) and (15) and recalling that $a_{1}^{(2)}=a_{1}^{(1)}$, $b_{1}^{(2)}=b_{1}^{(1)}$, we now have

$$
\begin{aligned}
& S_{\|}=-k^{3}\left\{a_{1}^{(1)}-b_{1}^{(1)}-n_{1}^{2}\left(a_{1}^{(1)}-a_{1}^{(3)}\right)+n_{2}^{2}\left(b_{1}^{(1)}-b_{1}^{(3)}\right)\right\}, \\
& S_{\perp}=k^{3} n_{1} n_{2}\left\{a_{1}^{(1)}-a_{1}^{(3)}+b_{1}^{(1)}-b_{1}^{(3)}\right\} .
\end{aligned}
$$

In terms of $S_{\|}$and $S_{\perp}$, the direct and cross polarized components of the back scattering cross section are

$$
\sigma_{\|}=4 \pi k^{-2}\left|S_{\| \mid}\right|^{2}, \quad \sigma_{\perp}=4 \pi k^{-2}\left|S_{\perp}\right|^{2} .
$$

It will be observed that only the direction cosines $n_{1}$ and $n_{2}$ appear in (46) and (47). If, therefore, the incident plane wave has either $\boldsymbol{E}^{\mathbf{i}}$ perpendicular to $\hat{\boldsymbol{z}}$ (so that $n_{\mathbf{1}}=0$ ), or $\boldsymbol{H}^{\mathbf{i}}$ perpendicular to $\hat{z}$ (so that $n_{2}=0$ ), $S_{\perp}$ is zero. This is a general result, true at all 
frequencies. In the particular case of axial incidence $\left(n_{1}=n_{2}=0\right)$,

$$
S_{||}=k^{3}\left(b_{1}^{(1)}-a_{1}^{(1)}\right) \text {. }
$$

This is the situation in which Siegel's empirical formula is relevant, and it implies

$$
\left|S_{||}\right|=k^{3} \frac{V F}{\pi},
$$

where $V$ is the volume of the cone and $F$ is the shape factor given in (2).

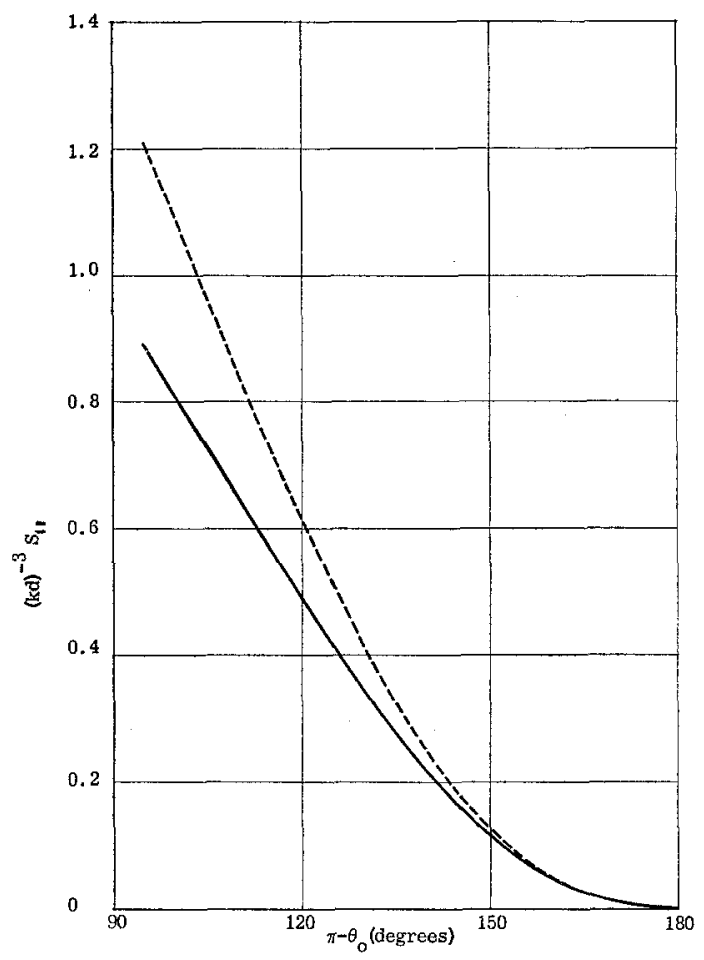

Fig. 3. Normalized back scattered amplitude for axial incidence: exact, - ; approximate (50), -----.

In Fig. 3 the exact values of $(k d)^{-3} S_{\|}$obtained from (49) are compared with the values predicted by (50), with the 'length-towidth' parameter $\tau$ in (2) taken as $\tau=\frac{1}{4} \operatorname{cosec} \theta_{0}$ (see [2]). It is observed that the empirical formula is remarkably accurate for 


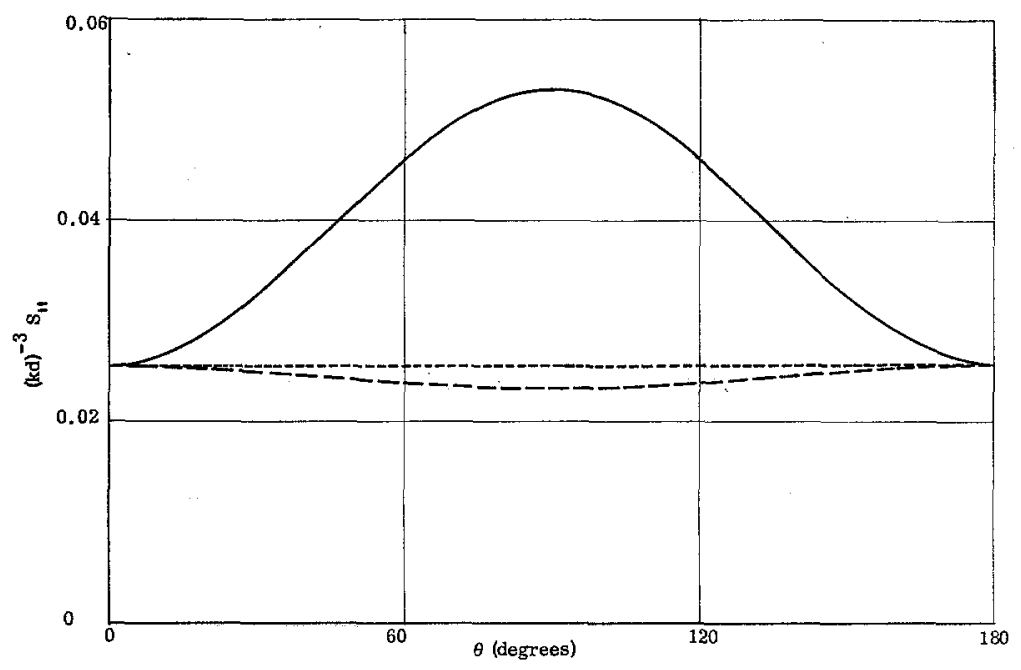

Fig. 4. Normalized back scattered amplitude for off-axis incidence on a 15 deg. half-angle cone:

exact (E-plane) —, $(H$-plane $)----$; approximate $(50), \ldots . . . .$.

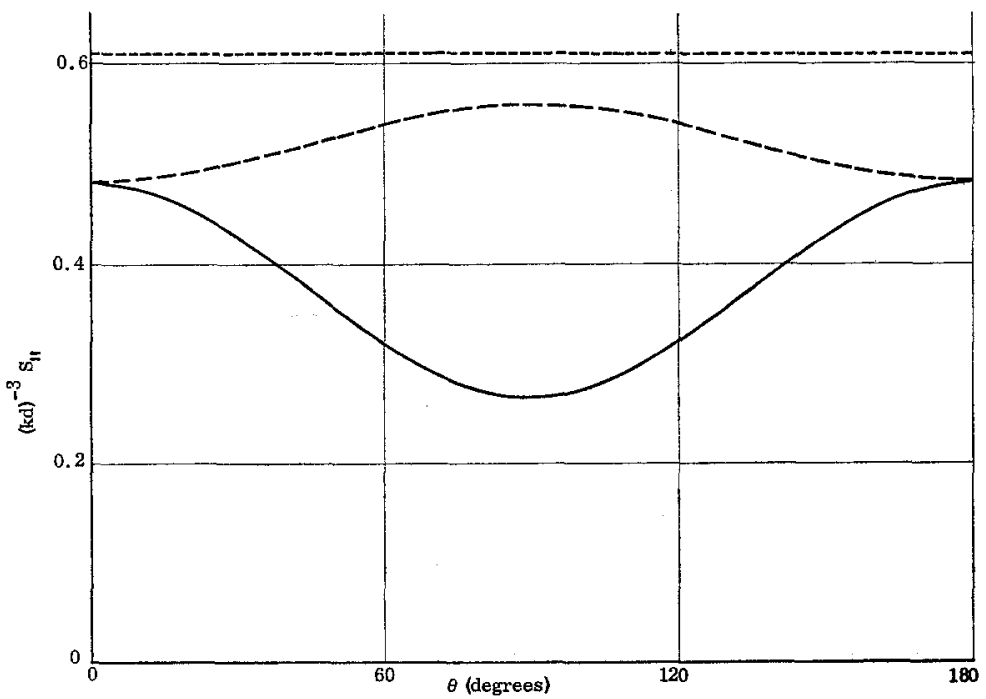

Fig. 5. Normalized back scattered amplitude for off-axis incidence on a 60 deg. half-angle cone: exact ( $E$-plane) $\longrightarrow,(H$-plane) -----; approximate $(50),-\ldots .--$. 
narrow angle cones, but overestimates the scattering by an increasing amount as $\theta_{0}$ decreases, and is in error by as much as $2.7 \mathrm{~dB}$ for $\theta_{0}=95 \mathrm{deg}$.

As noted in section 1, the empirical formula (50) is also widely used to estimate the scattering at all angles of incidence in spite of its inherent limitation to axial incidence and its consequent inability to predict the aspect variation of the back scattering pattern. To illustrate the error that is incurred thereby, we can use (46) to compute $(k d)^{-3} S_{||}$as a function of $\theta$ where $\theta$ is the angle from nose-on measured either in the $E$-plane $\left(n_{1}=\sin \theta\right.$, $\left.n_{2}=0\right)$ or the $H$-plane $\left(n_{1}=0, n_{2}=\sin \theta\right)$. The results for a narrow angle cone $\left(\theta_{0}=165 \mathrm{deg}\right.$.) are shown in F1g. 4 , and those for a wide angle cone $\left(\theta_{0}=120 \mathrm{deg}\right.$.) in Fig. 5 . For the $H$-plane patterns, the angular variation is relatively small, amounting to only about 10 percent for $\theta_{0}=165 \mathrm{deg}$. and 16 percent for $\theta_{0}=$ $=120$ deg., and in the latter case the estimate obtained from (50) is almost as close to the average as it is to the nose-on value. In the $E$-plane, however, the scattering is more aspect dependent, and significant errors could result from using (50) without regard to the aspect. This is particularly true for the wider angle cones for which $\theta=90 \mathrm{deg}$. is a minimum in the pattern, and the estimate obtained from (50) is too large even for $\theta=0$. In contrast, $\theta=$ $=90 \mathrm{deg}$. is a maximum for the narrow cone, with the situation reversed in the $H$-plane, and from an examination of (46) in conjunction with Fig. 2, it can be seen that for a cone having $\theta_{0} \simeq$ $\simeq 161 \mathrm{deg}$. the $H$-plane pattern is independent of aspect, whereas for a cone with $\theta_{0} \simeq 151 \mathrm{deg}$. the scattering in the $E$-plane is aspect independent.

\section{§ 5. Conclusions}

In spite of the relative simplicity of electromagnetic scattering problems at low frequencies, the number of finite bodies for which the low frequency scattering behavior is known precisely is still very limited, and it is because of this that the empirical formula (50) is so widely used.

A body of considerable practical interest is the round backed cone, and by means of a mode matching technique, we have determined the complete dipole moments from which all features of the low frequency scattering behavior can be deduced. Numerical 
results have been presented for a large range of cone angles. When the back scattering cross sections are compared with those predicted by (50), it is found that the latter is remarkably accurate for small-angle cones regardless of the aspect. For wide-angle cones, however, the empirical formula overestimates the nose-on cross section, and because of the greater aspect variation now displayed, significant errors could be incurred on using (50) to estimate the off-axis scattering.

\section{§ 6. Acknowledgements}

The author is indebted to Mrs. Austra Liepa and Mr. P. M. Wilcox for their computational assistance.

Received 24 October 1969

In final form 3 March 1970

\section{REFERENCES}

[1] Sifgel, K. M., Appl. Sci. Res. 7B (1958) 293.

[2] Sifgel, K. M., Proc. IEEE (Correspondence) 51 (1963) 232.

[3] LoRd Rayleigh, Phil. Mag. 44 (1897) 28.

[4] Rogers, C. C., J. K. Schindler and F. V. Schultz, The Scattering of a Plane Electromagnetic Wave by a Finite Cone, in Electromagnetic Theory and Antennas, Pergamon Press, New York 1963.

[5] Schultz, F. V., G. M. Ruckgaber, S. Richter and J. K. Schindler, The Theoretical and Numerical Determination of the Radar Cross Section of a Finite Cone, Purdue University School of Electrical Engineering Report No. TR-EE64-14.

[6] Kieinman, R. E, Proc. IEEE 53 (1965) 848.

[7] Wilcox, P. H., Math. Comp. 22 (1968) 205.

[8] Senior, T. B. A. and P. H. Wilcox, Radio Sci. 2 (1967) 479. 